\title{
A CONTROL SYSTEM FOR MODULATOR BASED ON ARM-LINUX
}

\author{
Guo Ji Hong \\ Radio and Television Digital Engineering Research \\ Center of Ministry of Education \\ Communication University of China \\ Beijing, China \\ Guojihong99@gmail.com \\ Zeng Zhi Bin \\ Radio and Television Digital Engineering Research \\ Center of Ministry of Education \\ Communication University of China \\ Beijing, China \\ zhbzeng@cuc.edu.cn
}

\begin{abstract}
China Mobile Multimedia Broadcasting (CMMB) is a self-developed wireless communication system for mobile terminals. The control board is the most important module in CMMB system. This thesis introduces a general modulator control system based on Arm-Linux platform. The platform of the system uses ARM chips as the master controller, by using the Linux embedded platform to achieve the internal among the control panel and baseband coding module, RF output module, RF processing module through the I2C bus, completes dynamic control of the modulator parameters. And LCD display based on Framebuffer, The control system has already been used in the CMMB modular, after testing, the system runs stable and reliable it is very versatility and practical
\end{abstract}

Keywords- Arm-Linux; FrameBuffer; $I^{2} C ; C M M B$;Control system;

\section{INTRODUCTION}

With the widely application of the ARM technology, in the embedded system, embedded processors that based on ARM microkernel, has been market mainstream. And the embedded Linux operating system is widely used because of its advantages about source and free, cutted kernel and function. At present, orienting embedded Linux system based on the ARM architecture has been becamed the product control system's mainstream choice. For thinking the performance, size and effect, this thesis is used core based on ARM and built an embedded Linux operating system on the processor, then to achieve the system control and human-computer interaction on the basis.

\author{
Li Zheng Xiang \\ Radio and Television Digital Engineering Research \\ Center of Ministry of Education \\ Communication University of China \\ Beijing, China \\ lizhengxiang@cuc.edu.cn \\ Zhou Shan Yi \\ Deloitte Touche Tohmatsu \\ University of International Business and Economics \\ Beijing, China \\ zhoushanyi1988@gmail.com
}

\section{SYSTEM STRUCTURE AND WORKING PRINCIPLE}

The design of control system based on Arm - Linux modulator framework is shown in figure 1. By Arm main control board, the baseband coding module, RF output module, RF four parts processing.

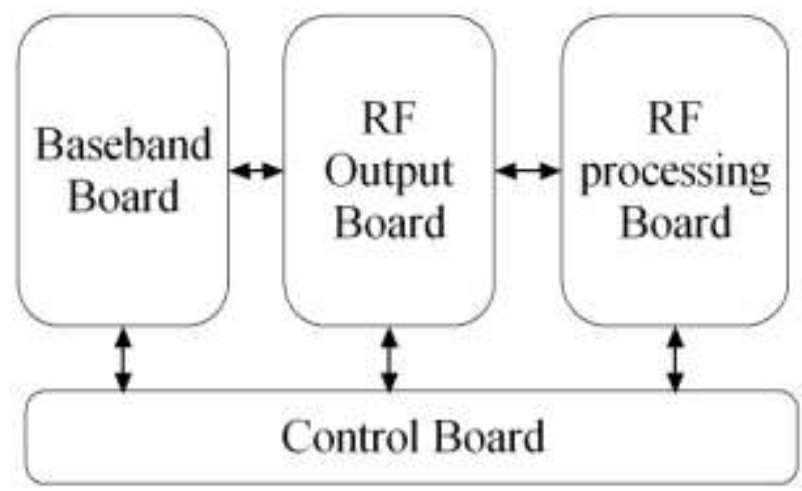

Figure 1 the overall structure of the equipment

Internal module used the $\mathrm{I} 2 \mathrm{C}$ communication protocol, complete equipment in the module parameters to pass through interrupts and FrameBuffer mechanisms to achieve a key control buttons and LCD display, complete the humancomputer interaction.

An external independent control by clicking the control panel keys to manipulate the upper applications, equipment parameter changes, control module the revised parameters, passed via the $\mathrm{I} 2 \mathrm{C}$ bus to the corresponding module, module control signal will be received after the printing adjustments was made, in order to complete the control of the device parameters to modify the Control Board. 
Actuator operating position, all the modules are running their own parameters via the $\mathrm{I} 2 \mathrm{C}$ bus back to the control module, control module receives the signal, that is printed at the LCD display, thus completing the controls on the device control panel.

\section{THE DESIGN AND IMPLEMENTATION OF THE SYSTEM}

\section{A. Control board hardware design and implementation}

Control system is the core package of equipment, in the process of designing the control panel, we must take into account all the necessary interfaces and chips, shown in Figure 2.

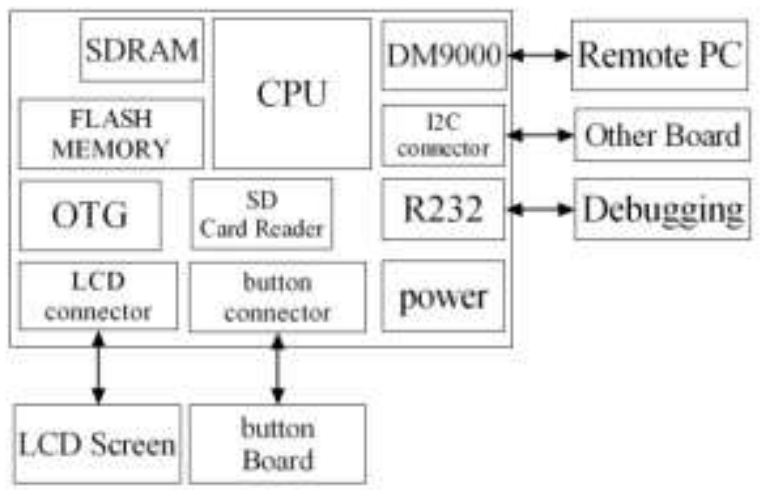

Figure 2 Structure of the control board

Network card chips are remote control for users at a later stage, so that users can remotely observing operation of the actuators. I2C interface is a Control Panel tool to communicate with other modules in order to achieve the dashboard control to other modules. R232 port is important while you are debugging interface. OTG interface allows users to quickly and easily through the PC USB interface for control system upgrade. SD card reader to provide dashboards with startup and fast burning system of multichannel programs with ease. LCD interface and button interface is for an external LCD screen and separate keys for the achievement of human-computer interaction.

\section{B. Internal Communications Based On $I^{2}$ C Bus}

I2C (Inter-IntegratedCircuit) bus developed by PHILIPS. There are two-wire serial bus for connecting the microcontroller and its peripherals.

I2C bus is a serial bus, which consist of SDA line and SCL line, it can send and receive data. I2C bus can send data between control board and the other three controlled board, its maximum transfer rate $100 \mathrm{kps}$. Various of circuits are connected in parallel on the I2C bus. And each circuit has a unique address. Each circuit is both a master and a controller, and it is both a transmitter and a receiver.

Bus transfers data in the process there are three types of signals, they are: the start signal, the end signal and response signal.

Start signal: SCL is high Voltage, SDA high level to low Voltage jumping, begun transmitting signals.
To signal the end of: the SCL to low Voltage, SDA from low Voltage to high Voltage jumping, end transmission

Response signal: after accepting the IC receives data to the 8bit data, IC issue that sends data to a specific low Voltage, says it has received data. Control boards after a signal from the Control Panel, wait for a response signal from the Control Panel, control panel after receiving the answer signal, continue to send signals according to the actual situation to make the judgment. If no answer is received a signal, is judged to be a control board failure.

Bus is controlled by a control panel. Control panels are used to produce the serial clock (SCL) hold the direction of transfer, and the start and stop conditions. (SDA) online data only can be changed during the SCL to low Voltage, SCL to high Voltage during the SDA state change is used to indicate the start and stop the conditions. As shown in Figure 3.

The control byte must be following the stat command, the top four is the identifier of device type(the different chip type have different definition, EEPROM is 1010 as usural), the next three are chip selection, and the last one is read \& write position, 1 means read operation, 0 means write operation. As shown in figure 3

\begin{tabular}{|c|c|c|c|c|c|c|c|}
\hline 1 & 0 & 1 & 0 & A2 & A1 & A0 & R/W \\
\hline
\end{tabular}

The Write operation cosists of byte write and paper write, and it's different of pape write by a load byte chip. The figure 4 shows the timing sequence of address, response and data transfer which is written by the peper write.

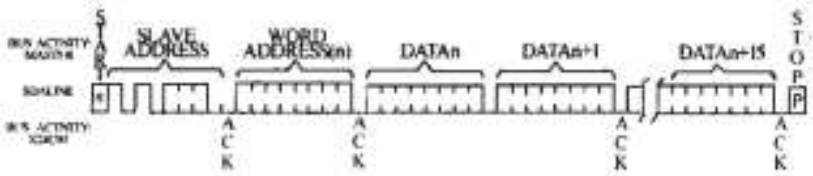

Figure 4 Page write operation timing diagram

Read operation contains three basic operation: the current address, Random read and sequential read. The figure 5 shows the timing sequence. It is should be noted that, the nine clock cycle of last read operation is not 'unconcern'. For shut down the read operation, the stop command or keeping SDA at high level must be send during the ,the nine clock cycle .

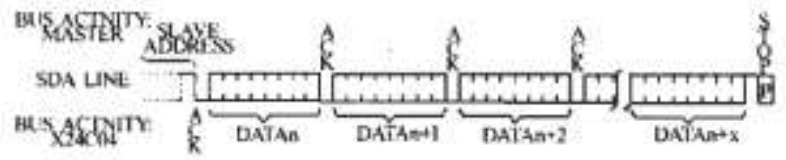

Figure 5 Sequential Read Timing Diagram

Communication system boards and blocks of the other modules with $\mathrm{I}^{2} \mathrm{C}$ communication protocol,actual implementation shown in Figure 6 below. 


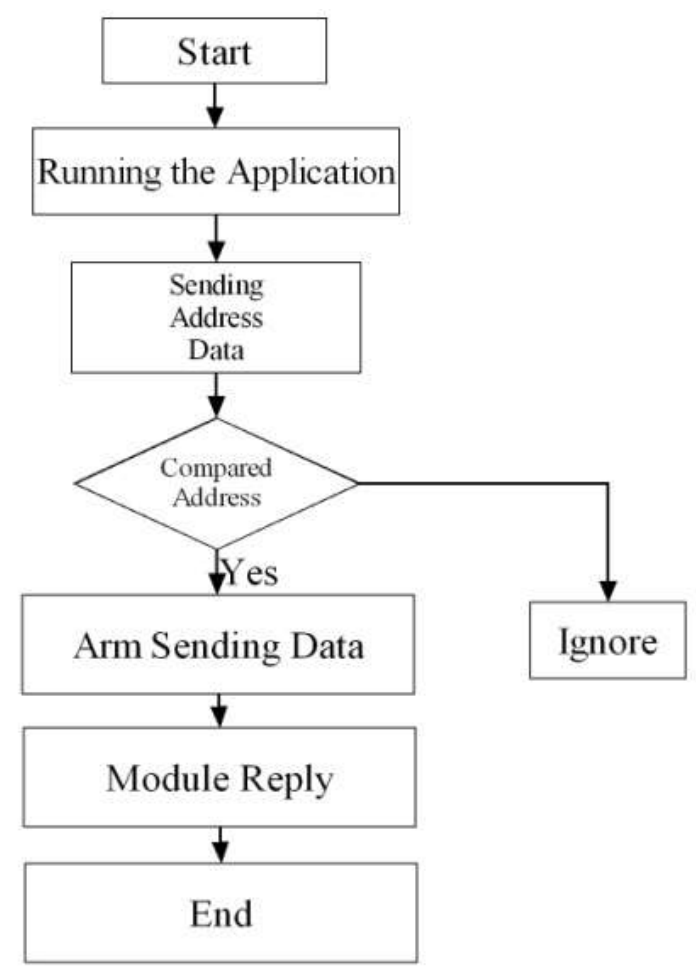

Figure $6 \mathrm{I}^{2} \mathrm{C}$ communication mechanism

Control Panel send 8-bit data at first, of which the first 7 bits represent the module address, 8th place indicates the direction of data transmission. If it is 0 , this is the Control Board to send traffic data, if 1 , this is communication module send data to the control panel.

Each module in actuator must have a unique address, three modules at the same time receive to 8-bit data, and then compared, if the address is ignored in this module that isn't the original address, if the module address, responding to an ACK signal, began internal communication.

In internal communication processes, control panel send the address to the called module and data one by one.

when the sending ended for CRC check. If the check code is same and then carries on the corresponding operation, if the check code is different to send data. It was shown in Figure 7.

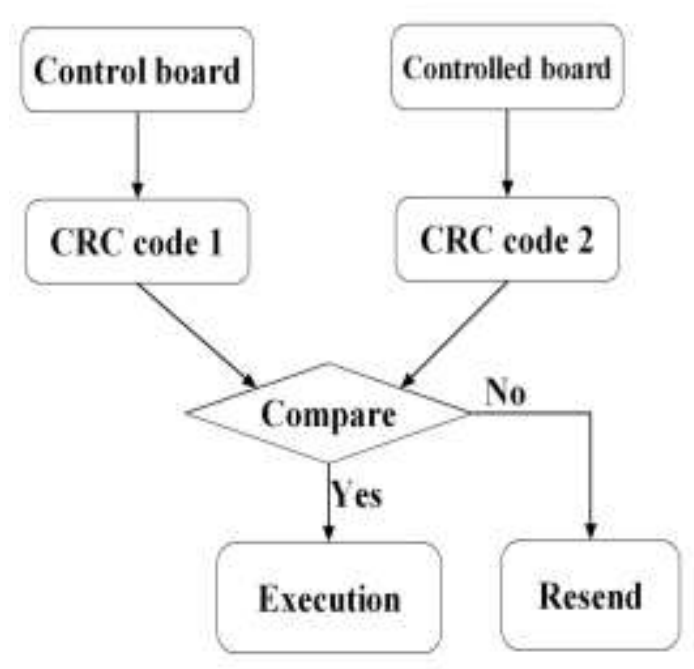

Figure 7 CRC checksum

Receive data after the dashboard to send communication modules to signal the end of $p$, completes this communication, namely, modifications of Exciter parameters.

\section{LCD Screen Display Based On FrameBuffer}

LCD screen is an important tool for people to interact with device, this design is based on the FrameBuffer.

FrameBuffer means frame buffer, it is a standard character device whose major device number is 29 , FrameBuffer mimics graphic capability that abstracting the graphic hardware structure into a series of data structures, we can directly operate the graphic card through FrameBuffer. Users can treat FrameBuffer as a memory display mapping, after mapping it to the processing address space, Reading and writing operations can be carried out directly and immediately displayed on the LCD screen, FrameBuffer interacting with the application is shown in Figure 8.

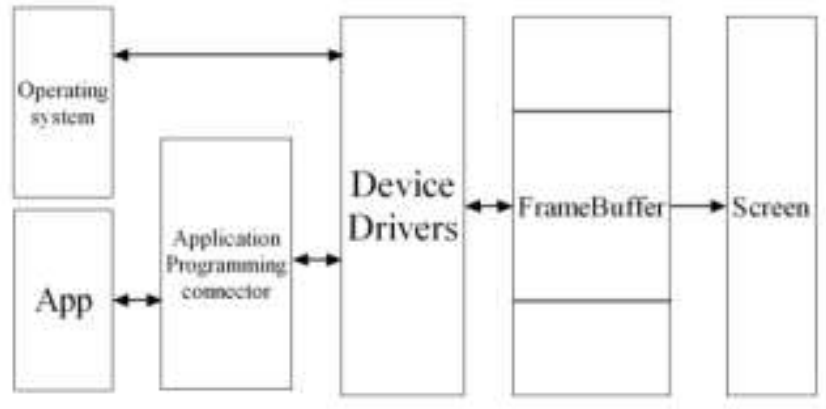

Figure 8 FrameBuffer interacting with the application

Most of the traditional LCD display use the method of setting the screen refresh time to updates, that is to set a refresh cycle, Once the counter reaches refresh cycle counter all data will be written to the mapped LCD virtual memory from actual memory actual instantly whether the user operate on the memory or not. The disadvantage is refresh cycle must be set to a small value to obtain real-time experience 
for users, meanwhile it is a waste of CPU resources writing the entire LCD screen, because the user control is usually causing modification and replacement of part of the lattice instead of the entire contents, and repeated operation take place in a very short time. This design use a real-time way of comparison literally to refresh the screen. Once the user operate actual memory will change, then, change the byte separately which is modificated and replaced. Greatly improving the user experience, as shown in Figure 9.

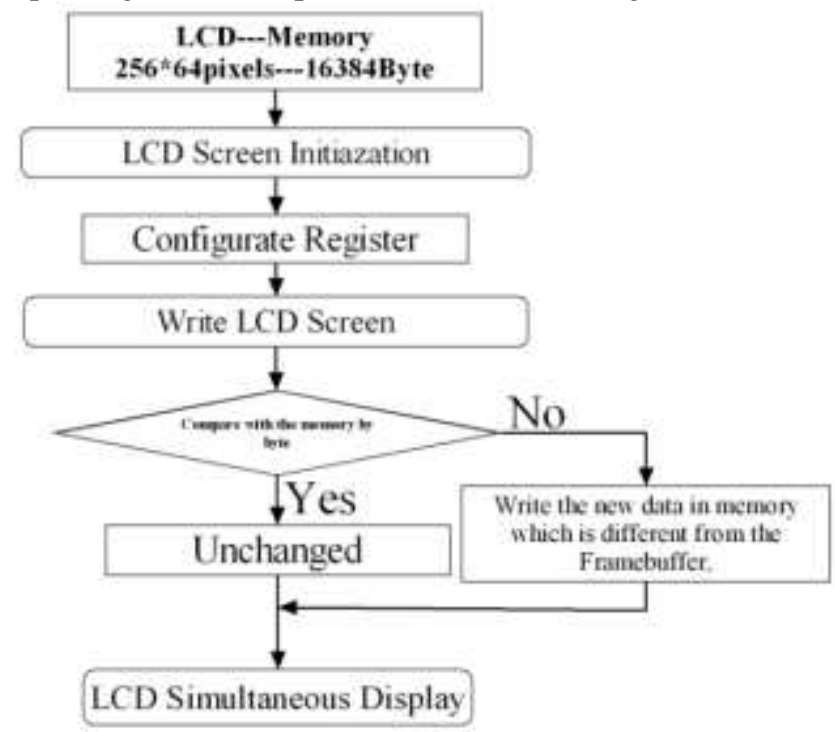

Figure 9 logic diagram of LCD driver implements

First, LCD constituting of $256 * 64$ pixels, each pixel composite from 2 color of black and white. So assign a size of 16384 bytes of memory as video memory. Then, LCD initialization, register a device to the system which major device number is 29 and minor device number is 4 . So that the system can detect a capacity of 16384 bytes of storage space. Then, register configuration, that is to configure the corresponding output through the operation readl and writel of LCD display control I/O, then read the data to the LCD mapped virtual memory from actual memory, finally, compare virtual memory and the actual memory one byte by one byte, if the byte is identical, there is no operation. If the byte is not the same, the data in actual memory will replace data in LCD mapped virtual memory, so that the LCD screen can be displayed in real time meanwhile reduce the CPU occupancy rate.

\section{CONCLUSION}

After commissioning of the overall actuator, the actuator can be found in accordance with the desired effect. The remaining three board can be perfect communicated with control board. At the same time, user can modify the data of the equipment by clicked on the button board, and LCD screen of actuator can display real-time job status perfectly. Perfect realization of human-computer interaction.

\section{REFERENCES}

[1] Li Wen, Cheng gengguo, and Xuan Zheng, "Research of image acquisition and decompressing based on ARM9 system," 622-625, 2010.

[2] Hu Zheng-wei, $I^{2} \mathrm{C}$ Protocol Design for Reusability, 2010, pp.83-86.

[3] Bruce,J.W.and Gray,M.A., "Personal digital assistant(PDA) based $\mathrm{I}^{2} \mathrm{C}$ bus anslysis," 2003, pp. 1482-1487.

[4] Miyawwaki,.K, "Evaluation of utility of the "Arm-Balancer" arm support system," 2010, pp 208-213.

[5] Righetti,X, "Proposition of a modular $\mathrm{I}^{2} \mathrm{C}$-based wearable architecture," 2010., pp 802-805.

[6] Yang Jia; Ji Wankang, and Hong Yongqiang, "Transplantation of GTK Based on FrameBuffer,” 2007,pp 2-381-2-384

[7] Ting Zhang, Chun Xu, and Yamin Yin "Design and implement of monitoring controller of TV transmitter on Arm-Linux",2010,pp 3134

[8] Zhihong Wang, Ruifang Liu , and Jie Liu "Development of Communication \& Storage System for NIR Spectrometer Based on Arm-Linux" 2010, pp 1281-1284

[9] Xingqiang Liu , Yuqing Huang, and Li Xu "Design for mobile node of sensor net works based on embedded Arm-Linux” 2008 ,pp 86018605

[10] Ping Li and Jian-Ping Li "Embedded intelligent home control system based on Arm-Linux" 2012 , pp 429-431

[11] R. Nicole, "Title of paper with only first word capitalized," J. Name Stand. Abbrev., in press.

[12] M. Young, The Technical Writer's Handbook. Mill Valley, CA: University Science, 